\title{
El debate sobre la identidad o identidades en Marruecos: Estratificación vs. Dinámicas de cambio
}

\author{
The Debate about Identity or Identities in Morocco: Stratification vs. \\ Dynamics of Change
}

\author{
Adil MOUSTAOUI SRHIR \\ Universidad complutense de Madrid \\ adil.moustaoui@pdi.ucm.es
}

Recibido: enero 2011

Aceptado: febrero 2011

\section{RESUMEN}

Este artículo analiza las dinámicas de cambio sociolingüísticas e identitarias en Marruecos y el debate que tiene lugar actualmente sobre a la cuestión de la construcción y legitimación de la identidad nacional marroquí. El análisis aborda la trascendencia del eje lengua e identidad y su relación con el modelo de Estado nación unitario, implantado en Marruecos desde la independencia hasta la actualidad. Del mismo modo, el artículo presenta un examen del paisaje identitario desde el punto de vista sociolingüístico y cómo dicho paisaje ha ido evolucionando a lo largo de los últimos cincuenta años, creando unas dinámicas de cambio en el mercado lingüístico e identitario en Marruecos. Por último, el artículo presenta un balance crítico del modelo de política lingüística e identitaria del Estado marroquí, proponiendo otro modelo acorde con estas dinámicas de cambio que están teniendo lugar actualmente.

El enfoque desde el cual se enmarca este análisis es el sociolingüístico crítico, por un lado, y el histórico-discursivo, por otro lado.

Palabras clave: Marruecos, Estado-nación, lengua, identidad, estratificación, dinámicas de cambio

\begin{abstract}
This article examines the sociolinguistic dynamics of change and identity in Morocco and the debate that takes place on the issue of the construction and legitimacy of the Moroccan national identity. The analysis focuses on the importance of language and identity axis and its relationship to the unitary nation state model implemented in Morocco since independence until now. The article presents also a review of landscape identity from the sociolinguistic point of view and how that landscape has evolved over the last fifty years, creating a dynamic market of change in language and identity in Morocco. Finally, the article presents a critical evaluation of the model of language policy and identity of the Moroccan State, proposing a different model according to these dynamics of change currently taking place. The approach from which frames this analysis is the sociolinguistic critic and historical discourse.
\end{abstract}

Key words: Morocco, Nation state, language, identity, Stratification, Dynamics of Change. 


\section{INTRODUCCIÓN}

La articulación del eje lengua, identidad y estado-nación fue y sigue siendo central en la creación y legitimación del Estado-nación, incluso, este eje todavía es clave en cómo se haya ido reproduciendo dicha legitimación en los distintos momentos de la historia de cualquier país o estado-Nación. Además, la lengua siempre ha jugado y juega un papel en la construcción de nuevas identidades y nuevas formas de pertenencia (ver Heller) ${ }^{1}$. No obstante, el capital lingüístico e identitario siempre ha estado relacionado en cómo se establecen las relaciones de poder, desigualdad y de diferencia en la sociedad y también con los cambios sociopolíticos y económicos. Concretamente estos cambios sociopolíticos son los que influyen, aunque fuese de manera indirecta, sobre la noción de Estado como estructura y como ideología, y por consecuencia, sobre las políticas lingüísticas y también sobre lo que Benjelloun ${ }^{2}$ denomina la politique d'identité del Estado. Marruecos, el objeto de estudio de este artículo, como cualquier Estado-nación ha cambiado y cambiaría a lo largo de su historia. Lo que nos interesa en el contexto actual es examinar como estos cambios a veces no deseados por el Estado, están repercutiendo en esa re-construcción y representación de lo que es Marruecos, lingüística, identitaria, étnicamente por parte del poder.

La importancia que empezó a tener la cuestión de la identidad o identidades de Marruecos dentro de esta dicotomía Unidad vs. Diversidad, no sólo en el discurso oficial dominante sino el resto de los discursos sociales y políticos, sean de contestación o de opinión, a raíz de las últimas propuestas de reforma del Estado marroquí para establecer su nueva re-organización política, administrativa y territorial, por una parte, y plantear sus reformas constitucionales, por otra parte, son una verdadera excusa para acercarnos a esta cuestión.

\subsection{EL OBJETO DE ESTUDIO}

En Marruecos, últimamente, se ha tratado la cuestión de la relación entre lengua e identidad en tanto que es un eje doble que fue y sigue siendo un tema fundamental en la mayoría de los discursos que se han producido en torno a la cuestión lingüística e identitaria, y no sólo en Marruecos, sino en el resto de los países del Magreb.

Pero, en primer lugar, hemos de señalar que los procesos identitarios se rigen como si fuesen un patrimonio nacional con un valor simbólico en la sociedad. En segundo lugar, cada individuo o grupo social concibe la identidad en función de sus convicciones, creencias y sus proyecciones imaginarias. Del mismo modo, las identidades son objeto de distintos paradigmas en la medida en que podríamos identificar algunos distinguiéndolos de otros, podríamos también situar a otros para excluirlos de un grupo o grupos, y dejarlos al margen de las normas establecidas dentro de un orden social determinado. Debemos señalar, por otra parte, que la

${ }^{1}$ HELLER, Monica, «Distributed Knowledge, distributed power: A Sociolinguistics of structuration», en Text \& Talk, $\mathrm{n}^{\circ} 27,5 / 6,(2007)$, págs.622-653.

${ }^{2}$ BENJELLOUN, Mohamed Othman, Projet national et identité au Maroc. Essai d'anthropologie politique, Casablanca, Eddif-L'Harmattan, 2002. 
identidad lejos de ser un ente es también un componente que genera debates y conflictos, a veces incesantes, entre distintos actores sociales.

Entonces, ¿cómo podemos concebir las relaciones entre las lenguas, las identidades, los procesos de cambio social en Marruecos? ¿En qué términos podemos analizar dicha relación? Y ¿por qué el eje lengua / identidad tiene mayor relevancia no sólo en el debate sobre las lenguas, sino el modelo de Estado-nación en general en Marruecos? ¿Cuáles son los factores que influyen en la continuidad, el cambio y la transformación de la identidad, en este caso, marroquí? ¿Y hasta qué punto estas dinámicas de cambio podrían generar cambio en la estructura y en las relaciones de poder existente en el mercado lingüístico y entre las diferentes identidades, por un lado, y en la creación de un nuevo modelo de gestión de los bienes simbólicos y materiales, por otro lado?

\subsection{MARCO TEÓRICO}

En primer lugar, este artículo parte de la noción de mercado lingüístico de P. Bourdieu $^{3}$. Y tal como la definió el sociólogo francés Bourdieu, existe una relación de poder entre las variedades lingüísticas consideradas bajas y con prestigio y el resto de las variedades lingüísticas con mucho valor tanto económico como social en el seno del mercado de los intercambios lingüísticos. Con lo cual, el campo lingüistico refleja en cierta medida las relaciones que existen entre las diferentes comunidades lingüísticas, donde cada comunidad es poseedora de un capital lingüístico que corresponde a su vez a una o unas identidades determinadas. De este modo, la situación sociolingüística se convertiría según $\mathrm{P}$. Bourdieu ${ }^{4}$ en un mercado cuyos productos o bienes simbólicos están expuestos bajo la forma de lenguas o variedades lingüísticas con sus correspondientes identidades, que están en una situación de competencia y dominio que "se manifeste par la position privilégiée qu'occupent les produits confortés par les rapports sociaux, notamment la politique culturelle et linguistique de l'État et/ ou le soutien des groupes économiques et socio-culturels dominants". (A. Boukous ${ }^{5}$ ) [el énfasis es nuestro].

No obstante, a pesar de este desequilibrio lingǘstico que se ve reflejado en lo económico, se produce en palabras de $\mathrm{P}$. Bourdieu "un système d'oppositions linguistiques socialement pertinentes tend à se constituer qui n'a plus rien de commun avec le système des oppositions linguistiques pertinentes linguistiquement", y que sigue operando en el mercado lingüístico. Dicho sistema de oposición podría tener distintas manifestaciones, en el caso que estudiamos veremos cómo se traduce mediante los procesos de continuidad y las dinámicas de cambio que se producen en campo lingüístico y en el seno de las identidades.

En segundo lugar, el artículo se enmarca dentro de la Sociolingüística Crítica "Dans le sens d'une sociolinguistique capable de révéler quels intérêts sous-tendent

\footnotetext{
${ }^{3}$ BOURDIEU, Pierre, “L'économie des échanges linguistiques". en Langue Française, no 34, (1977): págs. 17-34.

${ }^{4}$ BOURDIEU, Pierre, «Le fétichisme de la langue". En Actes de la Recherche en sciences sociales, $\mathrm{n}^{\circ} 4$ (1975), Paris, Maison des sciences de l'homme et l'École des Hautes Études en Sciences Sociales, pág. 4

${ }_{5}$ Boukous, Ahmed, Dominance et différence. Essai sur les enjeux symboliques au Maroc, Casablanca Editions Le Fennec, pág.11, 1999.

${ }^{6}$ Op.cit, 1975, pág. 4.
} 
les actions, les représentations et les discours, et qui bénéficie de l'évolution des processus sociaux" . El artículo parte también del estudio de la política lingüística tal como la define N. Labrie ${ }^{8}$, es decir:

«n'est pas uniquement la codification des pratiques langagières établie par des détenteurs du pouvoir (législatif, par exemple), mais bien l'ensemble des initiatives visant le contrôle social ayant pour objet le pluralisme et la variation linguistique provenant tant de la société civile que des institutions investies de pouvoir, avec tous les intérêts contradictoires que ces initiatives peuvent sous-tendre".

Cabe considerar que cualquier estudio del eje lengua/ identidad y política lingüística depende también de los cambios sociopolíticos y económicos más concretos, tanto a nivel nacional como internacional, que haya conocido nuestra área de estudio. Por ello, nuestro enfoque será también historicista.

En lo que concierne el concepto de identidad, nosotros partimos de la definición de Ph. Riley ${ }^{9}$ (2008: 87) que la define de esta manera:

"[...] we use 'identity' to talk about what makes individuals just that, individual. What makes 'me' me, as opposed to all other individuals, the agent of my actions, the continuing locus of my thoughts and memories, separately embodies in a numerically and physically distinct organism, which self-reports using the pronoun 'I', which is subjective and private and which has a proper name."

Por otra parte, consideramos que las identidades nacionales, concebidas como formas específicas de identidades sociales, étnicas, lingüísticas culturales y religiosas son discursivamente producidas, reproducidas y transformadas e incluso destruidas por medio del lenguaje o de otros sistemas semióticos (Wodak et al) ${ }^{10}$. Con lo cual, estamos de acuerdo con el enfoque planeado por H. Grad y L. Martín Rojo" que consideran que "Discourse analysis has shown how personal and social identities ara shaped in social interactions, and how they are created, reproduced, negotiated, imposed, or even resisted thouh discourse".

\section{ANÁLISIS DEL CONTEXTO}

\subsection{EL CONTEXTO SOCIO-POLÍTICO}

Si partimos del contexto social y político actual de Marruecos, y los cambios en todos los niveles que conoce, nos damos cuenta que estos factores desempeñaron y desempeñarían un papel primordial en este tradicional debate entorno al concepto de Estado, lengua e identidad. El mismo debate también nos muestra que a pesar de las relaciones de poder y desigualdad que existen entre el centro y la periferia, es

\footnotetext{
${ }^{7}$ HELLER, Monica, Eléments d'une sociolinguistique critique, Paris, L'Harmattan. (2003), pág. 10.

${ }^{8}$ LABRIE, Normand, "Les conflits linguistiques au Québec et au Canada: vers une grille d'analyse". En Labrie, N (dir): Etudes récentes en linguistique de contact, Bonn, Dümmler, (1997), págs. 213- 214.

${ }^{9}$ Riley, Philip, Language, Culture and Identity, New York \& London, Continuum, (2007), pág. 87.

${ }^{10}$ Wodak, Ruth y AL, "The discursive construction of national identity". en Discourse \& Society, Vol 10, n 2 (1999), SAGE Publications, London. págs. 149-173.

${ }^{11}$ Grad, Héctor y Martin Rojo, Luisa, "Identities in discourse: An integrative view", En Analysing identities in discourse, Dolón Rosana y Julia Todolí (ed), John Benjamins Publisching Company, Amsterdam \& Philadelphia, (2008), págs.8.
} 
decir, entre la identidad dominante e impuesta y las identidades in-visibilizadas, acabó convirtiéndose en una diálogo inter-discursivo de confrontación. Y no hace falta más que examinar las transformaciones discursivas (ver Moustaoui) ${ }^{12}$, tanto en el discurso oficial dominante como en el discurso alternativo periférico para comprobarlo. La representación que hacía el rey de Marruecos y de su identidad en el discurso del 31 de julio de 2001 que establece la creación del Instituto Real de la Cultura Amaziga no es nada comparable al discurso real del 6 de noviembre de 2009, en ocasión de la celebración de la fiesta de la Marcha Verde. Éste último discurso tampoco se podría equiparar con el discurso del 3 de enero de 2010 en el cual se hizo referencia a la creación de una comisión nacional para la regionalización. En este discurso el rey afirma lo siguiente:

$$
\begin{aligned}
& \text { "التشبث بمقدسات الأمة وثو ابتها، في وحدة الدولة والوطن و التراب، التي نحن لها ضامنون، و على صيانتها مؤتمنون. }
\end{aligned}
$$

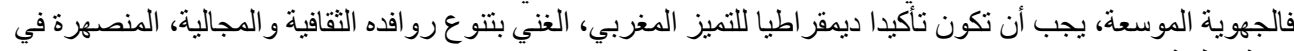

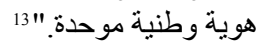

"La firmeza ante los valores sagrados y los pilares de la nación, notablemente la unidad del Estado y de su territorio, los cuales garantizamos y protegemos. Por lo tanto, la regionalización avanzada debería ser una confirmación democrática de la singularidad de Marruecos, rica por la diversidad de sus afluentes culturales y territoriales que se funden en una identidad nacional unificada." (Traducción del fragmento en árabe).

Este fragmento confirma el carácter unitario de esta construcción identitaria del discurso monárquico, siendo el discurso oficial con mayor legitimidad y autoridad en el país.

No obstante, el último discurso del rey pronunciado el 9 de marzo de 2011, como respuesta a las distintas manifestaciones convocadas en Marruecos en febrero y que exigían una urgente reforma constitucional y política es relativamente distinto en cuanto a representaciones de la identidad nacional marroquí. Dice el rey en este discurso:

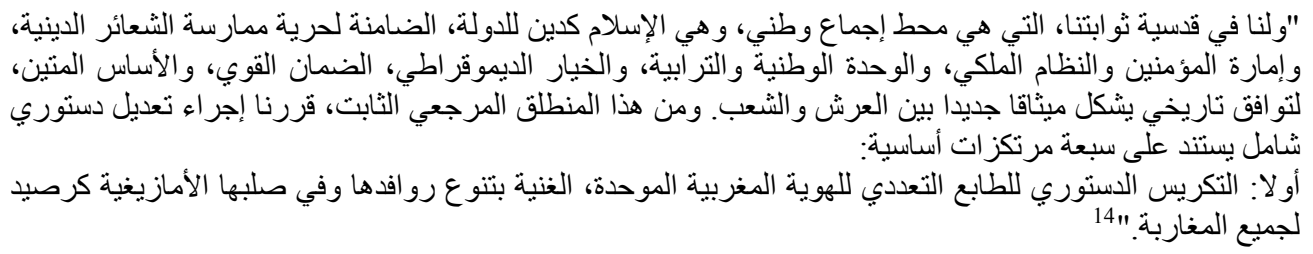

"La sacralidad de nuestros valores constantes, que gozan de unanimidad nacional a saber: el islam como religión del Estado el cual garantiza la libertad del ejercicio religioso; el Emirato de los creyentes; el sistema monárquico; la unidad nacional y territorial constituye la opción democrática. Todos ellos, son un sólido garante y una

\footnotetext{
${ }^{12}$ Moustaoui Srhir, Adil, Diversidad lingüistica, identidades y discursos en Marruecos. La pugna por la legitimidad, Berlin \& London, VDM-Publishing House, 2010.

${ }^{13}$ Discurso pronunciado por el Rey Mohammed VI, y dirigido a la nación marroquí el día 3 de enero de 2010 (disponible en internet en http://www.maroc.ma/NR/exeres/94BE45F6-4FDA-49AAB955-9ED04F7946AB,frameless.htm?NRMODE=Published)

${ }^{14}$ Discurso pronunciado por el Rey Mohammed VI, y dirigido a la nación marroquí el día 9 de marzo de 2011 (disponible en internet en http://www.maroc.ma/NR/exeres/187CF739-4DEA-47B1AD44-02D9A7EAFFAC.htm)
} 
potente base para un consenso histórico que representa un nuevo pacto entre el Trono y el pueblo. A partir de estos inamovibles referentes, hemos decidido llevar a cabo una revisión global de la Constitución, constituida sobre siete pilares fundamentales:

Primero: La consagración constitucional del carácter plural de la identidad marroquí unitaria y rica por la variedad de sus afluentes, donde la lengua amaziga ocupa un lugar central en tanto que patrimonio de todos los marroquíes." (Traducción del fragmento en árabe)

Se nota esta vez que la representación de la identidad cambia en cuanto a cómo se describe y se denomina ya que se introduce el carácter plural de esta identidad. No obstante, se mantiene la idea de que la identidad marroquí es única a pesar de que está compuesta por diferentes componentes, donde el amazigue ocupa un lugar central. Estas representaciones no se contradicen evidentemente con el modelo unitario de la gestión de la diversidad lingüística e identitaria que existe en Marruecos, ni con el resto de los modelos: el territorial, el político y el socio-económico.

Lo que es claro desde luego a través de este breve análisis de los discursos oficiales monárquicos, es que el concepto de identidad estatal, impuesto por el poder siendo la formación ideológica nacionalista más dominante, es considerado una forma de identidad colectiva; impuesta, imaginada y legitimada. Impuesta e imaginada por las acciones, y legitimada por las prácticas discursivas y legislativas que se enmarcan dentro de determinadas formaciones ideológicas. Es a partir de esta consideración que el Estado marroquí implantó su organización política, jurídica, administrativa y territorial desde la independencia hasta la actualidad.

Pero, si nos acercamos al discurso de contestación y que se opone al modelo estatal -el amazigue en este caso-, nos damos cuenta por ejemplo que las reivindicaciones expuestas en los Manifiestos, firmados por asociaciones y militantes amazigues, por la Autonomía del Rif, tanto del 18 de julio como del 7 noviembre de 2009 son diferentes a lo que se reivindicaba en la Carta de las Asociaciones Amazigues del Rif promulgada en agosto de 2005 que relamaba principalmente el reconocimiento del amazigue en la constitución marroquí. Lo mismo podríamos decir sobre cómo ha ido evolucionando el nacionalismo saharaui a favor de la independencia, tanto el producido por el frente interior, es decir dentro de Marruecos, como por el Polisario. Ambos discursos son manifestaciones claras de un nacionalismo regional o periférico, en el Rif o en el Sahara por ejemplo, basado en parte en el eje lengua / identidad. Dichas manifestaciones contestan y ponen en duda el proyecto nacional estatal y ponen en valor aspectos identitarios relacionados con la tradición y la base histórica de determinadas regiones, como hemos señalado anteriormente. Todos estos hechos nos demuestran que el poder estatal ha estado siempre inmerso en una confrontación sobre la cuestión del unitarismo frente al dualismo o el pluralismo, no sólo lingüístico, sino identitario con todo lo que implica el concepto de identidad.

Veremos ahora como se presenta en el momento actual esta base y este paisaje identitario en Marruecos, con especial énfasis en el plurilingüismo presente en este país. 


\subsection{PANORAMA IDENTITARIO ACTUAL EN MARRUECOS: DIVERSIDAD, CONTINUIDAD Y TRANSFORMACIÓN}

El hecho de identificarse el individuo con una lengua, una religión, un grupo, un pueblo o una nación no es, en la mayoría de los casos, un proceso consciente en el sentido de que no poseemos los rasgos que se atribuyen por una auto-identificación. Asimismo, asignarse una serie de rasgos o verse etiquetado por los demás son en realidad dos procesos distintos; no obstante, ambos están en una relación de interacción continua. Cualquiera que sea la naturaleza de los procesos identitarios, nunca son indefinidos; no obstante, están sujetos continuamente a modelos de referencia dominantes. Del mismo modo, las identidades se expresan de diversas formas en la medida en que cada individuo se caracteriza por una serie de rasgos sociales, culturales, lingüísticos y religiosos susceptibles de crear en él un sentimiento de pertenencia a grupos o comunidades determinadas, no forzosamente idénticas, pero distintas y diversas (Ver J. Edwards) ${ }^{15}$. A pesar de que el proceso identitario es complejo, nosotros intentaremos describir en el caso de Marruecos como se reflejan estas identidades y como se relacionan con las prácticas lingüísticas que son las que nos interesan.

Para nosotros, el estudio del proceso de afirmación de la identidad marroquí, con su evolución en función de las condiciones históricas y políticas y los cambios sociales que ha conocido el país, debería plantearse desde una perspectiva histórica y sociocultural, al mismo tiempo crítica. Este planteamiento ha de incluir, asimismo, no sólo el plurilingüismo en el que se basa, sino toda la historia social y política del país. Así, la identidad lingüística marroquí, es, según nuestra óptica, amaziga y árabomusulmana con toda la diversidad que supone ser amazigue o árabe o muslmán, también es francófona y mediterránea. (ver F. Laroussi 1997) ${ }^{16}$. Cabe señalar que esta descripción evidentemente es discutible si se plantea desde otro enfoque.

En lo que se refiere a la identidad desde el enfoque lingüístico, que es la que nos interesa en este estudio, según F. Laroussi' "La fonction identitaire d'une langue implique à la fois une diversité des formes et l'appropriation d'une ou de plusieurs formes linguistiques comme marque d'identification, et c'est en cela que l'identité nécessite la diversité: diversité de plusieurs langues différentes et diversité des formes d'une même langue". Segundo, cuando hacemos referencia a la relación entre lengua e identidad en Marruecos, debemos especificar a qué identidad nos estamos refiriendo y admitir al mismo tiempo el carácter diverso y múltiple de las identidades que comparte un individuo o un grupo social determinado en la sociedad marroquí. Y tercero, si el plurilingüismo es una realidad dada ya por existente en la sociedad marroquí, ello significa que las lenguas o las variedades lingüísticas remiten a identidades específicas y diferenciadas entre sí.

En primer lugar, la identidad marroquí es amazigue teniendo en cuenta el origen amazigue de la mayoría de los habitantes, incluso aquellos que no son amazigófonos.

\footnotetext{
${ }^{15}$ Edwards, John, Language and Identity, Cambridge, Cambridge University Press, 2009.

${ }^{16}$ Laroussi, Foued (dir), Plurilinguisme et identités au Maghreb, Rouen, Publication de l'Université de Rouen, 1997.

${ }^{17}$ Laroussi, Foued, "Minoration linguistique au Maghreb : langues, nations et identités". En Revue DiversCité langues, Vol VII (2002), Montréal, Université de Québec, pág.7.
} 
En segundo lugar, los hablantes del amazigue, en términos sociopolíticos se consideran un grupo étnico, aunque no es mayoritario, y en términos sociolingüísticos, es considerado una agrupación lingüística. Estos dos factores han hecho que a lo largo de la historia, la identidad amaziga esté presente y se construya en función de las circunstancias sociopolíticas por las que ha pasado el país. A nivel socio-discursivo, cabe destacar que el discurso alternativo amazigue se basó y se sigue basándose en la identidad amaziga en su proceso de reivindicación de sus derechos lingüísticoculturales, y también en la argumentación del modelo sociolingüístico y de política lingüística que propone para el estado marroquí y que contra-argumenta en algunos aspectos el modelo estatal. Del mismo modo, el discurso oficial reconoce la particularidad amazigue de Marruecos tanto a nivel étnico, lingüístico y social, a pesar de que su representación de lo amazigue siempre se ha limitado al hecho de que sólo forma parte de una identidad nacional marroquí única y unificada.

El segundo elemento de la identidad marroquí es el árabo-musulmán. El lazo de unión que se ha creado por la presencia árabe del norte de África con la coiné árabe y el Islam hizo que se crease un proceso identitario que se apoyaba principalmente en el árabe como lengua y en el Islam como religión. Este contacto con la civilización árabe y el Islam se podría considerar como uno de los factores que generaron la reivindicación de la identidad árabo-musulmana, no solamente en Marruecos sino en todo el Magreb, a pesar de que ningún marroquí o magrebí tiene al árabe clásicoestándar como lengua materna. En este proceso, cabe señalar el papel que ha desarrollado la ideología panarabista dominante que ha tenido una percepción negativa sobre las lenguas vehiculares maternas y las lenguas occidentales en la medida en que se las consideraban como elementos que fomentaba la división, y por lo tanto, constituían una amenaza para la unidad de la nación árabe-musulmana, una nación, que en cierto modo es ficticia. Dice E. Abulkacem- Afulay ${ }^{18}$ que

« Si le nationalisme, dans sa version première, était fondamentalement territorial, ayant pour vocation la constitution en entité politique - couvrant le territoire précis de l'Empire chérifien - de la culture savante liée à l'Islam, son contenu idéologique et politique a subi, après la deuxième guerre, une profonde transformation. L'accent est désormais mis sur la langue comme principe unificateur et sur la propagation de la croyance en une origine commune, en conformité avec l'origine de l'ethnie arabe. Ainsi, les marqueurs identitaires courants dans la définition substantialiste de l'ethnie commençaient à structurer le champ des représentations élaborées à propos de la nation marocaine. »

Este proceso identitario histórico de la construcción y legitimación de la identidad árabe tiene pues como base la religión musulmana, y como referente lingüístico y cultural la variedad clásica-estándar. Asimismo, se apoya en la política lingüística de arabización y está respaldada por el discurso público dominante y oficial. Ello, genera un proceso de minorización y de estigmatización del resto de las variedades lingüísticas -en concreto amazigue y árabe marroquí- y por supuesto,

${ }^{18}$ Abulkacem- Afulay, El Khataria, "Formation discursive nationaliste et arabité du Maroc", en Revue Asinag, $\mathrm{n}^{\circ} 1$ (2008), págs.59-60. 
de las identidades que representan. A este propósito $\mathrm{F}$. Laroussi ${ }^{19}$ señala que "Lorsque l'arabité est en prise directe avec l'islamité, c'est souvent l'identité arabe qui est mise en avant". De este modo, la identidad árabo-musulmana, al estar definida en relación con la religión, que en este caso es un referente simbólico con poder y legitimidad, y con la lengua oficial, en este caso, el árabe, se la presenta como nacional, única y legítima. Es, pues, en este contexto ideológico-político donde se definió la identidad nacional y en consecuencia el marco constitucional e institucional relacionado con las lenguas y sus hablantes en la sociedad marroquí, tras la independencia del país. Un referente apoyado y adaptado también por los movimientos nacionalistas que terminaron por imponer la identidad árabe como norma dominante en la construcción del Estado-nación marroquí independiente ${ }^{20}$.

Por otra parte, cabe destacar que el nuevo discurso dominante institucional empezó a incluir la identidad Afro-sahariana como un componente más de la identidad marroquí. Esta inclusión del elemento saharaui en los discursos oficiales del rey, se debe evidentemente al conflicto del Sahara occidental presente en el debate no sólo territorial y político sino identitario en Marruecos. Una identidad saharaui que se ha ido construyendo a lo largo de la historia política y social de Marruecos en este espacio territorial y también lingüístico ya que la variedad lingüística del Hasaniya juega también un papel clave en este proceso de construcción. A ello sumamos la evolución histórica de la estructura étnica, social y política de las tribus saharauis, que hizo que esta identidad saharaui estuviera mucha más presente, explícita y en continua evolución y representación. (A. Hernández ${ }^{21}$. Asimismo, el factor identitario como hemos visto en los últimos acontecimientos que tuvieron lugar en el Sahara, desempeñó y seguirá desempeñando un papel clave en el surgimiento del nacionalismo saharaui que reclama una independencia total de Marruecos.

La tercera identidad presente en Marruecos sería la francófona puesto que el francés es lengua institucional sin ningún estatus jurídico. Pero hablar de la identidad francófona en Marruecos no es tan fácil como puede parecer, teniendo en cuenta las relaciones complejas que unen Francia con el Magreb. Primero, cabe destacar el pasado colonial todavía no enterrado; segundo, la cuestión de la independencia y la construcción de un estado-nación marroquí con todo lo que implica, y tercero la confrontación que existe entre modernidad y tradición, un factor que ha reforzado la tendencia a reivindicar una identidad árabo-musulmana y marroquí sin ningún referente francófono u occidental. No obstante, en este contexto y viendo el estatus de la lengua francesa, a pesar de ser la lengua del colonizador, como lengua de apertura a la modernidad, a la tecnología y al mundo occidental y el papel que desempeña en tanto que lengua enseñada y de enseñanza, lengua de poder y también viendo la valoración de los marroquíes del francés, que

\footnotetext{
${ }^{19}$ Op.cit. 2002, 8

${ }^{20}$ Para profundizarse más en la relación que hay entre el nacionalismo árabe y la lengua ver Suleiman, Yasser, The arabic language and national identity, Washington, Georgetown University Press, 2003.

${ }^{21}$ Hernández, Angela, "Territorio, historia e identidad: Sus El Aksa o Sáhara occidenta", en Revista de Estudios Internacionales Mediterráneos, $n^{\circ} 9$ (2010), Madrid, TEIM, págs. 26-36.
} 
en general, es positiva (A. Boukous 200022). Esta visión positiva está relacionada principalmente con las exigencias del mercado sociolaboral y con las posibilidades que ofrece el hecho de ser francófono en Marruecos para poder acceder a los recursos económicos y ascender socialmente. Pero la cuestión que se plantea es la siguiente: ¿es realmente el francés una seña de identidad de los marroquíes francófonos? Y lo es de tal modo qué podemos hablar de una identidad francófona en Marruecos o en el Magreb. Sobre esta cuestión F. Laroussi ${ }^{23}$ opina que:

"Être ou ne pas être francophone ne signifie pas parler ou ne pas parler « bien » le français, mais surtout se l'approprier. Le français n'est une marque identitaire que dans la mesure où à terme il se créolisera. Pour devenir réellement une forme identitaire au Maghreb, le français doit s'adapter et se transformer. Il serait alors paradoxal de vouloir à la fois défendre la place du français dans le monde et continuer à mener une politique conservatrice prônant la défense de la norme menacée du sacro-saint bon usage”.

Según nuestra opinión, para llegar a este hecho la noción de la francofonía ha de conocer un cambio rápido en su génesis; asimismo, su concepción ha de dejar de ser impuesta por la ideología francesa jacobina, y ofrecer, así, un margen para una reivindicación de una identidad francófona con todas sus particularidades, tanto en Marruecos, como en el resto de los países considerados francófonos. Este hecho podría ser posible una vez que se proceda a lo que F. Laroussi ${ }^{24}$ llama magrebizar el francés dándole un valor no solamente lingüístico sino cultural, local y también nacional.

Otra dimensión de la identidad marroquí que podríamos destacar, sería en este caso, la mediterránea debido al contacto que hubo y que hay entre Marruecos y el resto de las culturas mediterráneas. El espacio mediterráneo compartido entre Marruecos y el resto de los países de la zona propicia hoy en día un intercambio cultural, lingüístico, económico y social. Todos estos factores han hecho que parte de la población marroquí se identificase como mediterránea, introduciendo, de esta manera, otra identidad en la sociedad marroquí, a pesar de que esta identificación no está relacionada con ningún elemento lingüístico sino la suma de muchos componentes. A ello, añadimos la inclusión de este componente mediterráneo de la identidad marroquí en el discurso monárquico, aunque no de manera frecuente.

\section{IDENTIDADES EN MARRUECOS: JERARQUIZACIÓN Y ESTRATIFICACIÓN}

El proceso de transformación de Marruecos tras la independencia, de un Estado tradicional, caracterizado por una diversidad étnica, lingüística y cultural e identitaria, hacia un Estado unificado simbólicamente e ideologizado a nivel de su nacionalidad, conllevó a la creación de un nacionalismo cuyo objetivo era imponer una unidad socio-

${ }^{22}$ Boukous, Ahmed, "Les Marocains et la langue française". En La coexistence dans l'espace francophone: approche macro-sociolinguistique. Actes de deuxième Journées Scientifiques de réseau de l'AUF, Paris, AUPEL, UREF, 2000, págs. 13-30.

${ }^{23}$ Op.cit. 2002, 9

${ }^{24}$ Op.cit. 2002,9. 
cultural (Fischman ${ }^{25}$ ), y añadimos aquí el componente lingüístico e identitario a esta noción de unidad socio-cultural. Pero, a nivel lingüístico e identitario, la cuestión es diferente en este proceso de transformación. Porque dentro de esta nacionalidad marroquí unificada e ideologizada existen unas variedades lingüísticas correspondientes a diferentes comunidades expandidas e impuestas socialmente, pero marginalizadas y no reconocidas oficial y políticamente como identidades propias tal como hemos visto en los últimos discursos del monarca. Dichas comunidades, no sólo representan elementos étnicolingüísticos e identitarios claros sino que podrían representar hasta regiones bien separadas históricamente en su organización a todos los niveles. A pesar de esta unidad ideologizada y simbólica, la presencia de estas comunidades es la que genera diferencias a nivel, regional e incluso local, y nacional. Entonces, si el sentimiento y la conciencia de pertenecer a una identidad, ya sea étnica, cultural regional, o nacional, es el principal promotor de la construcción del nuevo estado-nación independiente después de la independencia, constatamos que este mismo modelo es el responsable de la existencia de una jerarquización en la política lingüística e identitaria llevada a cabo por el estado desde la independencia hasta la actualidad. Por lo tanto, el proceso de reconocimiento oficial de Marruecos de una lengua o variedad y su plasmación mediante políticas, sean educativas, lingüísticas, identitarias y sociales está ligado directamente al reconocimiento de la identidad que representa dicha lengua. Hasta el momento, el discurso dominante oficial y también el legislativo reconocen una sola identidad árabe ligada a la oficialidad única de la lengua árabe ${ }^{26}$. Este reconocimiento genera, por un lado, una exclusión desde lo oficial y nacional a nivel macro-político y social de la identidad amazigue. También, notamos que la realidad social y lingüística árabe en Marruecos se caracteriza por la presencia de una diversidad de identidades, y una diversidad, incluso lingüística, teniendo en consideración la importancia de la variación lingüística árabe que está presente en el espacio lingüístico marroquí. Dicho de otra manera, lo árabe en Marruecos, hasta el momento ha generado dos identidades; una identidad árabo-musulmana vinculada al árabe clásico; siendo ésta última la lengua del Corán, una identidad lingüística y cultural relacionada con el papel del árabe estándar en el mundo arabófono como lingua franca de comunicación supranacional y como elemento asimismo de unión, unificación y de modernidad e independencia; de la misma manera, ha generado el desarrollo de una identidad árabe-marroquí, que con el paso del tiempo y los cambios sociales, políticos y económicos se ha ido construyendo y ampliándose, creando sub-identidades regionales y locales como es el caso de cualquier habla o variedad regional del árabe marroquí o del Hasaniya en el Sahara. Pero dicha identidad macro, étnica, lingüística y regional ha estado mitigada y subordinada a las dos identidades anteriores dominantes. De esta forma, la subordinación simbólica del árabe marroquí como variedad lingüística y como elemento cultural fue un factor que generó: i) su exclusión total del discurso dominante oficial puesto que no se le considera como una entidad, ii) la población marroquí, en general, o los actores sociales, en particular, que están en contra de la arabización como política

${ }^{25}$ Fischman, Joshua, "Nationality-Nationalism and Nation-Nationism", en FISHMAN, Joschua; FERGUSON, Charles; DAS GUPTA, Jyotirinsdea (dir): Language Problems of Developing Nations; New York, John Wiley \& Sons, págs. 39-51, 1972

${ }^{26}$ Ver Preámbulo de la constitución marroquí. 
lingüística, educativa y cultural, incluyen todo lo árabe con todas sus connotaciones, sea la dominante, representada por el árabe clásico-estándar, sea la subordinada que representa el árabe marroquí. Dicho hecho, justifica el porqué se está retrasando el reconocimiento político y jurídico del árabe marroquí como entidad propia y como variedad lingüística que pueda ser el instrumento de comunicación a nivel institucional en el seno de la sociedad marroquí, así como la lengua de la modernidad. Todos estos factores intervinieron de manera directa en el tipo de políticas lingüísticas que se pusieron en marcha en Marruecos después de su independencia, y también en el tipo de relaciones que se establecieron, no solamente entre las diferentes comunidades lingüísticas y sus identidades correspondientes, sino también entre las distintas prácticas discursivas producidas por dichos grupos sociales, relacionadas con la cuestión de la lengua y la identidad. Unas relaciones que se caracterizan por una clara estratificación social y política de las lenguas y las identidades en Marruecos.

\section{LAS DINÁMICAS DE CAMBIO SOCIOLINGÜÍSTICAS E IDENTITARIAS: HACIA UN NUEVO MODELO}

Para nosotros la identidad, tanto si es cultural, social o religiosa, como si es lingüística, es un proceso en continua evolución, transformación y emancipación que depende del tipo de las relaciones sociales que lo determinan y que se establecen entre los diferentes grupos étnicos. Así, una vez que se cambien estas relaciones, cambiarán de igual forma las actitudes hacia las variedades lingüísticas, los grupos de hablantes y étnicos, y hacia todas las referencias identitarias relacionadas con estos componentes. La compleja noción de identidad, siendo un elemento en continua evolución y cambio nos incita a afirmar que todas las dinámicas y manifestaciones giran a su alrededor; sean políticas, económicas o sociales, son susceptibles hacia el cambio.

Pero no cabe duda de que el eje central y la base de todas estas dinámicas de cambio social, político, económico, incluyendo las distintas formas de representar e imaginar a Marruecos discursivamente, tiene relación con como va cambiando la distribución del capital y los recursos en el mercado de bienes simbólicos y materiales, y el reparto de poderes y la desigualdad. Tienen asimismo relación con cómo se van rompiendo relaciones de subordinación entre las lenguas, las comunidades lingüísticas y los grupos sociales.

En el momento actual, el primero ejemplo claro que refleja estas dinámicas de cambio es el proceso de re-valorización y reivitalización del amazigue en el campo lingüístico. Sobre esta cuestión Boukous ${ }^{27}(2008: 21$ y ss) señala que:

"La situation de l'amazighe va connaître un changement qualitatif á partir des derniéres decennies dans un contexte politique national et international marqué par la culture des droits de l'Homme. [...] Suivant ce référentiel, six arguments principaux fondent la légitimité de l'amazighité:

\footnotetext{
${ }^{27}$ Boukous, Ahmed, “Le champ langagier: diversité et stratification”, en Revue Asinag, nº 1(2008), págs. 21-ss.
} 
Elle est une donne historique qui plonge ses racines au plus profond de l'histoire et de la civilisation maghrébines;

Elle constitue un èlément essentiel de la culture et du patrimoine commune a toutes les composantes de la communauté nationale sans exclusive;

Sa promotion constitue un levier important dans le projet de société démocratique et moderniste auquel aspire le Maghreb;"

No cabe duda de que todos estos factores conllevan a un proceso de emancipación lingüística e identitaria de la comunidad amazigófona. En dicha emancipación entra en juego también cualquier proceso de emancipación social y económica que tiene relación con la gestión de los recursos y al acceso al conocimiento, desde el nivel más local hasta el nacional.

El segundo ejemplo, es el empoderamiento lingüístico, social y político que está conociendo últimamente el árabe marroquí. En los últimos años se ha extendido el uso del árabe marroquí a otros ámbitos en los cuales se usaba antes el árabe estándar o francés, es el caso de la prensa escrita, la radio y la televisión, incluso la literatura. Asimismo, ahora en algunos ámbitos de carácter institucional se usa el árabe marroquí; por ejemplo en algunas intervenciones de ciertos miembros del Parlamento, en el discurso político propagandístico, y el discurso de algunos medios de comunicación. Por otra parte, hemos de señalar un dato muy significativo que tiene que ver con el aumento de la demanda para aprender árabe marroquí o el aumento de programas de alfabetización en dicha lengua. Todas estas prácticas lo que hacen realmente es romper con las relaciones históricas de diglosia y de subordinación que se han mantenido entre las variedades estándar, árabe o francés, y árabe marroquí. Dichas prácticas vienen apoyadas por un discurso en un contexto de cambios sociales y políticos que coincide con una clara evolución del uso y estatus del árabe marroquí en Marruecos. Con lo cual, no es una casualidad que se produzca este empoderamiento por una parte, y este discurso social que lo apoya, por otra parte.

Esta nueva realidad sociolingüística, relacionado con la emancipación lingüística de dos variedades lingüísticas que históricamente han sido subordinadas, está generando nuevas concepciones sobre la propia identidad y unas nuevas formas de pertenencia, sea a un grupo social, la comunidad lingüística o una región o territorio determinado con todos sus componentes. Dice Laraoui ${ }^{28}(2009)$ :

"Existen varios y diversos asuntos, ocurridos o previstos, que tienen relación con los derechos colectivos. Desde la lengua hasta la distribución de los bienes, pasando por el sistema educativo y la organización. Hoy día, dichos asuntos parece difícil resolverlos puesto que se plantean a nivel central. Como este nivel no está lo suficientemente homogenizado, entonces llegar a un consenso sobre ello sería difícil. Es por este motivo que se evita debatirlo en el seno del Parlamento y del Gobierno y se deriva al máximo árbitro, es decir, al rey. Este hecho en concreto es el que conlleva a una interpretación de la constitución, encaminada hacia la opinión reservada, es decir, hacia el Estado unitario. Lo apropiado ¿no sería poner desde el primer momento estos asuntos en manos de los propios interesados, aquellos que sufren daños por dichos asuntos y los usan como argumentos?" (Traducción nuestra)

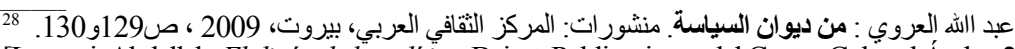

[Laraoui, Abdellah, El diván de la politica, Beirut, Publicaciones del Centro Cultural Árabe, 2009, pág. 129-130] 
La cita de Laraoui encaja bien con la opinión de un campesino amazigue que pertenece a una pequeña aldea situada a las afueras de Méknes, una región históricamente amazigófona. Dice el campesino refiriéndose a su propia identidad:

"Yo soy amazigue, soy de aquí de Aït Amer. Mi vida es mi terreno y el día día"29

Por lo tanto, esta Triangulación entre las formaciones discursivas, sea la oficial y la alternativa- que representan distintos movimientos de contestacion social-, ya que ambos son dominantes y el discurso del campesino amazigue, nos sugiere lanzar una serie de preguntas: ¿Qué relación existe entre el Estado y la identidad? ¿Existe realmente un modelo equitativo, democrático y moderno para la gestión del capital, sea el material (recursos) o el simbólico (lengua, identidad, cultura) a nivel de todas las escalas señaladas arriba?

Lo cierto es que generalmente cualquier acción política, sea jurídica, administrativa, educativa, lingüística o cultural nunca toma en consideración la opinión de los interesados ni la micro-pluralidad existente dentro de la diversidad, sea en Marruecos o en otros países. Pero lo cierto también, es que en Marruecos últimamente todas las iniciativas de carácter no estatal de desarrollo y de distribución de los recursos de manera equilibrada se hacen a nivel local. Para nosotros, estos cambios en la gestión del capital, generó, genera y generaría una diversidad identitaria que va cambiando y aumentando con el cambio del contexto social, político, económico e ideológico. Como consecuencia, las políticas lingüísticas e identitarias deberían superar este enfoque globalizador y pasar a lo que A. Boukous ${ }^{30}$ propone como solución a la estratificación lingüística existente en el campo de los bienes materiales y simbólicos en Marruecos, es decir, la glocalisation. Esta perspectiva para nosotros supone lo siguiente:

i) una nación moderna e independiente y con una diversidad, como es el caso de Marruecos, debería no presentar e imponer en las políticas, sean lingüísticas o identitarias un elevado grado de unidad sociocultural, incluyendo, la unidad lingüística e identitaria;

ii) el estado debería reconsiderar la nacionalidad como una entidad sociocultural, con el resto de los componentes-lingüísticos e identitarios-, que no corresponde a una realidad geográfica y política de un Estado-nación; iii) desatar el nacionalismo-sea el dominante o el regional-de los elementos ideológicos y emocionales que tienen una relación directa con "una única nacionalidad", "una única nación", "una única lengua" y "una única identidad".

Estas propuestas conectan el proceso identitario con el nivel micro puesto que concibe la identidad en términos comunitarios, es decir, que cada comunidad sea étnica, lingüística o social dispone de una propia identidad. Del mismo modo, este modelo descentralizaría las políticas lingǘsticas e identitarias en la medida en que reconoce no sólo la diversidad existente sino la pluralidad de esta diversidad. Por último, este modelo supone pues que la diversidad, sea lingüística o identitaria, es un beneficio político, social y económico para el Estado y la ciudadanía en el nivel local, regional y nacional, e incluso interacional. Con lo cual, su reconocimiento sería un paso hacia el establecimiento del Estado moderno, democrático y equitativo.

${ }^{29}$ Entrevista realizada en mayo de 2010.

${ }^{30}$ Op.cit, 2008. Pág.34. 